\title{
Nonaxisymmetric Shapes of a Magnetically Levitated and Spinning Water Droplet
}

\author{
R. J. A. Hill* and L. Eaves \\ School of Physics and Astronomy, University of Nottingham, Nottingham NG7 2RD, United Kingdom
}

(Received 1 August 2008; published 1 December 2008)

\begin{abstract}
The shape of a weightless spinning liquid droplet is governed by the balance between the surface tension and centrifugal forces. The axisymmetric shape for slow rotation becomes unstable to a nonaxisymmetric distortion above a critical angular velocity, beyond which the droplet progresses through a series of 2-lobed shapes. Theory predicts the existence of a family of 3- and 4-lobed equilibrium shapes at higher angular velocity. We investigate the formation of a triangular-shaped magnetically levitated water droplet, driven to rotate by the Lorentz force on an ionic current within the droplet. We also study equatorial traveling waves which give the droplet threefold, fourfold, and fivefold symmetry.
\end{abstract}

DOI: 10.1103/PhysRevLett.101.234501

PACS numbers: 47.55.D-, 24.10.Nz, 97.60.Lf

In experiments published in 1863, Plateau devised a way to study the behavior of liquids in the absence of gravity by suspending olive oil in a density-matched water-alcohol mixture [1]. The shape of a spinning oil droplet, driven by a rotating shaft, became flattened at the poles as the droplet gained speed, while the equatorial diameter increased. Beyond a critical angular velocity, the droplet evolved into a spinning nonaxisymmetric shape resembling a triaxial ellipsoid, which developed into a two-lobed shape with increasing speed. Plateau was inspired by the idea that the droplet's surface tension could model the influence of self-gravitation on spinning astronomical bodies [2]. It was later realized that the droplet model could provide insights into the behavior of atomic nuclei $[2,3]$.

Although the simplicity of Plateau's technique for studying weightless fluids on Earth is attractive, comparison of the results with theory is complicated by shape deformations due to the viscous drag of the surrounding fluid. Here we avoid the problem of drag by using a $16.5 \mathrm{~T}$ verticalbore, superconducting magnet to diamagnetically levitate droplets of water [4-8], with volumes up to $6 \mathrm{ml}$, in air. The droplet is spherical at rest; the measured polar (vertical axis) to equatorial diameter ratio is $1.00 \pm 0.02$. Beaugnon et al. have already demonstrated the usefulness of magnetic levitation for studying the dynamics of weightless droplets [6]. We have combined magnetic levitation with a "liquid electric motor" spinning technique, to observe the following new features in the dynamics of a spinning droplet: (i) a triangular rotating shape closely related to 3-lobed equilibrium shapes considered theoretically, (ii) large-amplitude waves with up to 5 nodes, traveling around the droplet's equator. We pass an ionic current $I$ through the droplet by means of two thin, parallel, gold wire electrodes spaced $d=4.0 \mathrm{~mm}$ apart [9]. One wire is aligned with the bore axis and the droplet center, so that the current flows perpendicular to the magnetic field $(B)$ vector. The resulting Lorentz force generates a torque $\tau=$ $B I d^{2} / 2$ on the droplet, causing it to rotate around the vertical axis through the droplet's center (Fig. 1 and Fig. S1 [9]).

Brown and Scriven investigated the equilibrium shapes of a rigidly rotating droplet theoretically using finite element analysis, grouping the shapes into "families" according to their symmetry [10]. The 2-lobed shape family, which includes the ellipsoidlike shapes observed in Plateau's experiments and 2-lobed "peanut" shapes observed in orbiting spacecraft $[11,12]$ and in rolling "liquid marbles" [13], branches from the axisymmetric family when the dimensionless angular velocity $\Omega^{\star}=$ $\sqrt{\rho \Omega^{2} R^{3} / 8 \sigma}$ reaches $\Omega_{\text {II }}^{\star}=0.56$. Here $\Omega, \rho$, and $\sigma$ are the angular velocity, density, and surface tension of the droplet, respectively, and $R$ is the radius of the spherical droplet at rest $[10,14]$. A 3-lobed and a 4-lobed family branches from the axisymmetric shapes at $\Omega_{\mathrm{III}}^{\star}=0.71$ and $\Omega_{\mathrm{IV}}^{\star}=0.75$, respectively, but Brown and Scriven concluded that these shapes should be unstable to small shape perturbations and thus be unobservable. However, experiments on acoustically levitated liquids showed that a $\sim 1 \mathrm{~mm}$-diameter droplet with a 3-lobed shape could be
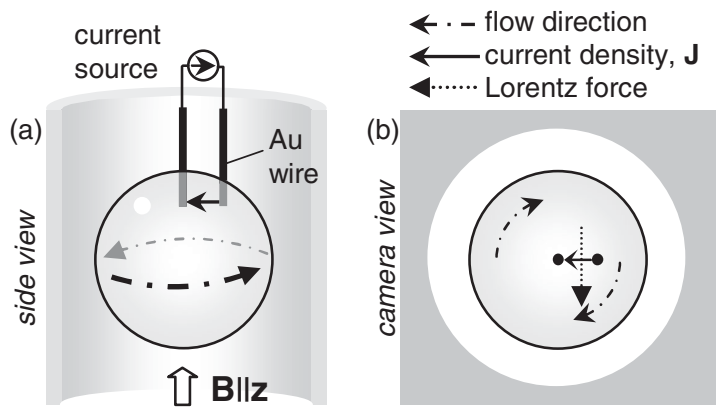

FIG. 1. Schematic of a spinning, levitating droplet inside the magnet bore viewed from (a) side and (b) below magnet (camera view). The electrode positions, electric current, and Lorentz force are indicated. 
stabilized if forced into large periodic oscillation [15], but the droplet dynamics were far from equilibrium.

Our technique generates a surface wave that travels around the droplet's equator in the opposite direction to the spin. By exciting a small-amplitude traveling wave with 3 nodes, we are able to suppress the degeneration of the triangular equilibrium shape (a member of the 3-lobed shape family) into the 2-lobed shape. This is similar to the stabilization technique used in Ref. [15], but in our method the amplitude of the surface wave is small compared with the droplet size, so that the equilibrium shape is observed clearly.

We first describe observations of a three-lobed equilibrium shape in a droplet with volume $V=1.5 \mathrm{ml}(2 R=$ $14 \mathrm{~mm}$ ) to which a small quantity of surfactant is added, reducing $\sigma$ from 74 to $34 \pm 2 \mathrm{~mJ} \mathrm{~m}^{-2}$. We increase $\tau$ gradually by increasing $I$ at a constant rate from $I=0$ to $I=600 \mu \mathrm{A}$ in $1 \mathrm{~min}$ and then hold $I$ steady to maintain a constant $\tau$. The spinning droplet is axisymmetric, bulging at the equator and flattened at the poles. As it picks up speed, the equatorial radius $r$ increases. Figure S2 [9] shows the variation of $r$ and $\Omega$ with time $t$ and $I(t)$.

When $\Omega$ exceeds $\sim 2$ revolutions per second (rps), the equatorial outline becomes slightly elliptical [Fig. 2(b)]. This shape does not rotate with the flow of the liquid but remains fixed relative to the position of the off-axis electrode. Then, $20 \mathrm{~s}$ later, a 2-node oscillation develops, traveling azimuthally around the elliptical outline at $2.5 \pm$
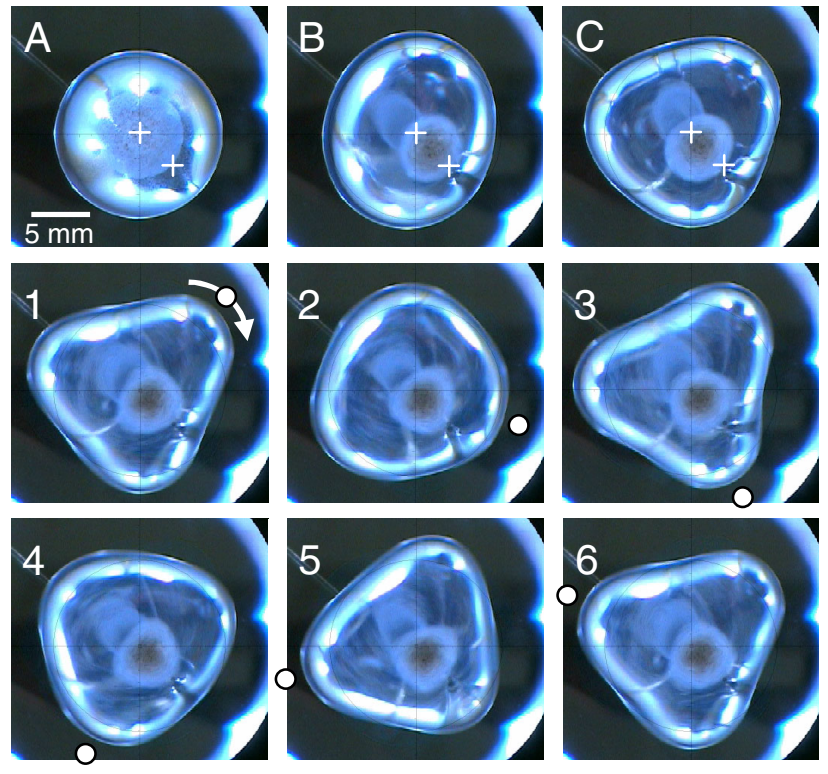

FIG. 2 (color online). A levitating $1.5 \mathrm{ml}$ droplet. (a) Not rotating. (b) Liquid rotating at $\Omega / 2 \pi \sim 2.0$ rps: the droplet's equator has an elliptical outline. (c) 2.5 rps: the equator has the symmetry of an equilateral triangle. In (b),(c) the outline is not rotating with the fluid. Crosses show the electrode positions. (1)(6) Consecutive movie frames, $40 \mathrm{~ms}$ apart; here, the triangular outline is rotating with the fluid in the arrow's direction at $\Omega / 2 \pi=3.33 \mathrm{rps}$. White circles follow one corner.
$0.1 \mathrm{rps}$ (movie 1 [9]). However, less than $10 \mathrm{~s}$ after the current reaches $600 \mu \mathrm{A}$ the equatorial outline of the droplet develops the threefold symmetry of an equilateral triangle. This outline remains fixed relative to the position of the off-axis electrode [Fig. 2(c) and movie 2 [9]]. Approximately $10 \mathrm{~s}$ later, a 3-node oscillation develops, moving around the equator, over the surface of the static shape, in the same direction as the flow of the liquid (movie 3 [9]). The oscillation amplitude increases during the next few seconds, while the amplitude of the lobes of the static shape on which it is superposed dies away. Once the static shape has decayed, approximately $5 \mathrm{~s}$ after the onset of the oscillation, the droplet has a regular triangular outline which rotates at $\Omega / 2 \pi=3.33 \pm 0.05 \mathrm{rps}$ [see Fig. 2 (1)-(6) and movie 4 [9] ]. Close to the axial electrode the fluid is rotating faster, at $\sim 5 \mathrm{rps}$, as is evident from the vortex of small bubbles liberated by hydrolysis, and there is additional vorticity generated at the off-axis electrode. Away from the electrodes, however, the frequency with which tracer particles orbiting at a radius $\sim 0.3 R$ from the axis perform a complete revolution around the droplet agrees well with the angular velocity of the droplet outline (Fig. S2 [9]). Thus we can describe the rotation of the droplet as rigid body-like, in the sense that the fluid has a constant "background" vorticity $\xi=2 \Omega$ (like that of rigid rotation) plus some localized vorticity generated by the electrodes.

Immediately after the onset of the rotating triangular shape we observe a decrease in the rotation rate of the fluid in the axial vortex, indicating a transfer of angular momentum from the vortex to the rest of the fluid (Fig. S2 [9]). Similar behavior is observed at the onset of the 2-node oscillation during spin-up. The rotating triangular shape remains stable for $\sim 100$ revolutions before the rotation becomes eccentric (camlike).

The equatorial shape of the droplet following the onset of the triangular shapes is well approximated by

$$
r(\phi, t)=R\left[1+a+b_{W} \cos (m \phi)+b_{R} \cos (m(\phi-\Omega t))\right]
$$

representing two superposed oscillations with the same wave number $(m=3)$, similar maximum amplitudes, $b_{W}(\max ) \approx 0.3$ and $b_{R}(\max ) \approx 0.3$, but differing frequencies. Here $\phi$ is the azimuthal angle, increasing clockwise in Fig. 2. The $b_{W}$ term represents the static triangular shape and the $b_{R}$ term represents the clockwise-rotating triangular rigid-body-rotation (RBR) shape. [Equation (1) also describes the static elliptical shape with superposed 2node oscillation, observed during spin-up, if we set $m=$ 2.] In the frame of the rotating droplet, the static shapes are revealed to be azimuthally traveling waves, with frequency $\omega=m \Omega$, excited by the action of the off-axis electrode moving relative to the fluid. The droplet outline in the rotating frame is given by 


$$
\begin{aligned}
r\left(\phi^{\prime}, t\right)= & R\left[1+a+b_{W} \cos \left(m\left(\phi^{\prime}+\Omega t\right)\right)\right. \\
& \left.+b_{R} \cos \left(m \phi^{\prime}\right)\right],
\end{aligned}
$$

where $\phi^{\prime}=\phi-\Omega t$. Comparison of the $b_{W}$ term in Eqs. (1) and (2) demonstrates that the static shapes observed in the laboratory reference frame are observed as traveling waves in the rotating frame. We shall always use the phrase "static shape" to mean a shape that does not rotate in the laboratory reference frame (i.e., not rotating in the video images), and similarly for "rotating shape." We emphasize that the static shape is generated from a surface wave moving relative to the fluid.

As the wave amplitude $b_{W} \rightarrow 0$, the kinematics tend to that of RBR and we can compare our results with theory developed for equilibrium RBR shapes. Theory predicts a bifurcation point where 3-lobed equilibrium shapes may develop from the axisymmetric equilibrium shape at $\Omega_{\text {III }}^{\star}=0.71$ [10]. The triangular rotating shape appears at $\Omega^{\star}=0.75 \pm 0.05$, in good agreement with theory. Although $b_{W}$ is small, so that the kinematics are close to $\mathrm{RBR}$, it does not vanish completely $\left(b_{W} \sim 0.1 b_{R}\right)$; the influence of the traveling wave is just discernible in Fig. 2 as a small periodic oscillation in the amplitude of the lobes of the rotating shape. Since the traveling wave is "attached" to the off-axis electrode, a wave mode with $m$ nodes is excited only at a particular angular velocity $\Omega=$ $\omega / \mathrm{m}$. In this $1.5 \mathrm{ml}$ droplet, the condition to excite $m=3$ waves coincides with the theoretically predicted 3-lobed bifurcation point $\Omega_{\mathrm{III}}^{\star} \approx 0.71$. We observe the stable 3-lobed RBR shape only for a limited range of $R$ and $\sigma$. This suggests that, while theory shows that the equilibrium of 3-lobed RBR should be unstable to 2-lobed shapes, the 3-lobed RBR shape is stabilized by interaction with this small-amplitude $m=3$ traveling wave. In acoustic levitation experiments on smaller $(10 \mu \mathrm{l})$ droplets, Ohsaka and Trinh [15] obtained stabilization of a 3-lobed shape by exciting the axisymmetric $l=2, m=0$ spherical harmonic, but due to the relatively large amplitude of this oscillation, the kinematics in their experiments are far from RBR. The 3-lobed and 4-lobed rotating shapes have been observed in spinning droplets suspended in densitymatched liquids, but in this case the shapes are deformed considerably by viscous drag [16]. Our measured critical angular velocity values for 3-lobed bifurcation are summarized in Table I, along with corresponding data for the 2-lobed shape, for comparison.

We now describe the excitation of azimuthally traveling waves with up to 5 nodes in $1.5-6.0 \mathrm{ml}$ droplets without

TABLE I. Measured and theoretical angular velocities at 2-

\begin{tabular}{|c|c|c|c|}
\hline \multirow{2}{*}{$\begin{array}{l}\text { Shape } \\
\text { Family, } n\end{array}$} & \multicolumn{2}{|c|}{ Measured } & \multirow{2}{*}{$\begin{array}{c}\text { Theory } \\
\Omega_{n}^{\star}\end{array}$} \\
\hline & $\Omega / 2 \pi(\mathrm{rps})$ & $\Omega_{n}^{\star}$ & \\
\hline 2-lobed & $2.5 \pm 0.1$ & $0.57 \pm 0.05$ & 0.56 \\
\hline 3-lobed & $3.33 \pm 0.05$ & $0.75 \pm 0.05$ & 0.71 \\
\hline
\end{tabular}
and 3-lobed bifurcation point for $1.5 \mathrm{ml}$ droplet. surfactant added. We detail the behavior of a $3 \mathrm{ml}(2 R=$ $18 \mathrm{~mm}$ ) droplet as a representative example. We increase $I$ to $600 \mu \mathrm{A}$ in $1 \mathrm{~min}$, then by increasing $I$ slowly, at $20 \mu \mathrm{A} / \mathrm{min}$, from this point, we can observe the 2-node oscillation evolve over approximately 2 min into a (nearly) rigidly rotating shape resembling a triaxial ellipsoid (movies (6)-(8) [9]) with major axis perpendicular to the rotation axis and $\Omega / 2 \pi=2.6 \pm 0.1 \mathrm{rps}\left(\Omega^{\star}=0.58 \pm 0.03\right)$. When $I$ reaches $660 \mu \mathrm{A}$ the amplitude of the 2-lobed rotating shape decays and the droplet takes on the threefold symmetry of an equilateral triangle [Fig. 3(a) and movie 9 [9] ]. When $I$ is kept constant at this point, the triangular static shape persists for over $3 \mathrm{~min}(\sim 500$ revolutions) before an instability to strongly eccentric rotation causes the drop to break free of the electrodes. However, when we increase $I$ to $680 \mu \mathrm{A}$, the outline spontaneously develops a fourfold (square-shaped) symmetry; this shape is also fixed relative to the electrodes [Fig. 3(b) and movie 10 [9] ]. The square shape persists for approximately $10 \mathrm{~s}(\sim 30$ revolutions), until the outline of the drop gains yet another corner, taking on the fivefold symmetry of a static regular pentagon [Fig. 3(c) and movie 11 [9] ]. Approximately $10 \mathrm{~s}$ later, the shape transforms into a 2-lobed spinning "peanut" in under $2 \mathrm{~s}$ [Fig. 3(d) and movie 12 [9]]. For this droplet, the triangular, square, and pentagonal shapes appear at $\Omega_{m} / 2 \pi=2.7 \pm 0.1,2.9 \pm 0.1$, and $3.1 \pm 0.2 \mathrm{rps}$, respectively (Fig. S3 [9]). These shapes are azimuthally traveling waves in the reference frame of the rotating

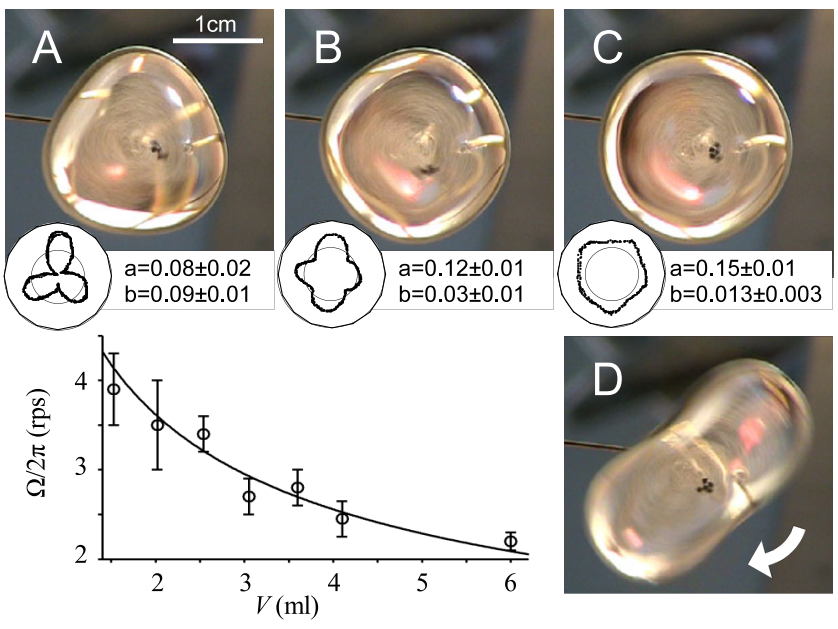

FIG. 3 (color online). (a)-(c) $m=3,4$, and 5-node waves traveling around the equator of a levitating $3 \mathrm{ml}$ droplet, rotating at $\Omega / 2 \pi \sim 3$ rps. The wave nodes remain fixed in the camera's view since the wave's angular velocity $\omega / m$ is equal to $\Omega$ but opposite in direction. Insets: polar plots of the amplitude of the equatorial bulges $\Delta r(\phi)=r(\phi)-R \approx R[a+b \cos (m \phi)]$, where $r(\phi)$ is the equatorial radius and $\phi$ is the azimuthal angle. Each plot's outer radius represents $2 \mathrm{~mm}$ amplitude. (d) Clockwise rotating 2-lobed "peanut" shape, rotating at $\Omega / 2 \pi=1.70 \pm 0.04 \mathrm{rps}$; major axis length $3.0 \pm 0.2 \mathrm{~cm}$. The graph shows measured values of $\Omega$ at the onset of the $m=3$ wave as a function of droplet volume $V$. Solid line: wave angular velocity $\omega / m$ of $m=3, l=3$ normal (Rayleigh) mode. 
droplet, with $m=3,4,5$. The equatorial droplet radius is well approximated by Eq. (1) with $b_{R}=0$; measured values of $a$ and $b=b_{W}$ are given in Fig. 3.

The graph in Fig. 3 shows the measured angular velocities at the onset of the $m=3$ wave, $\Omega_{3}$, for seven droplets with volume $V=1.5-6.0 \mathrm{ml}$. The data are well represented by $\Omega_{m}=\omega_{0} / m$, where $\omega_{0}=[l(l-1) \times$ $\left.(l+2) \sigma / \rho R^{3}\right]^{1 / 2}$ is the frequency of the normal mode (Rayleigh) oscillation [17] and $l=|m|=3$. Theory predicts $\omega(m, l)=\omega_{0}(l)+(m / l) \Omega$ to first order in $\Omega[18,19]$, giving $\Omega_{m}=\omega_{0} /(m-1)$ for $\Omega \ll \omega$ (since $l=|m|$ ), in contrast to our result. Since $\Omega$ cannot be considered a small perturbation in our experiments [the equilibrium droplet shape is far from the spherical shape assumed in the $O(\Omega)$ theory] it is not surprising that an $O(\Omega)$ approximation does not fit our data. Nevertheless, it is interesting that the data suggest $\omega$ is unaffected by the droplet's spin in these experiments. Experimentally determined $\Omega_{4}$ and $\Omega_{5}$ are in reasonable agreement with $\omega_{0} / m$ for $m=4$ and 5 [9], although the agreement is not as clear as for $m=3$. The frequencies of the $l=2, m= \pm 2, \pm 1,0$ spherical harmonics at higher rotation rates have been investigated using numerical methods [18], but the calculations for the $|m|=3$ modes have yet to be attempted. A nonlinear theory of large-amplitude traveling waves, on the scale observed in our experiments, has been developed for nonrotating droplets [20] but the case of rapid rotation has not been studied to our knowledge. Previously, traveling waves with $m=2$ and the related tesseral oscillation $(l=2, m=$ \pm 1 ) have been observed using acoustic levitation [21,22]. The experiments presented here represent the first observation of $|m|>2$ traveling waves in a weightless liquid droplet, to our knowledge.

The behavior of a liquid droplet has proven to be an effective way to gain an intuitive understanding of the behavior of objects on much larger (astronomical) and smaller (nuclear) scales. The theoretical stability of the shapes of spinning, self-gravitating astronomical objects follows a similar pattern to that of spinning droplets [23] and recent studies of the light curves of Kuiper-belt objects have identified several rapidly spinning bodies that are likely to have a triaxial shape due to their large angular momentum [24]. The event horizon of the black hole has been described as a membrane endowed with surface tension [25]. It has been proposed [26,27] that in a higher dimensional space-time the horizon may become unstable to lower-symmetry shapes analogous to the nonaxisymmetric droplet shapes we observe. The liquid droplet model of atomic nuclei and the shape instabilities of a spinning droplet has recently stimulated investigation of the Jacobi shape transition in rapidly rotating atomic nuclei, for which there is some experimental evidence ([28] and references therein). We suggest that the experimental results presented here could stimulate further insights.

We acknowledge M. Swift, A. Padilla, and E. Copeland for useful discussions. This project is supported by a Basic
Technology Grant from EPSRC, UK; Grant No. GR/ S83005/01.

*richard.hill@nottingham.ac.uk

[1] J. Plateau, Annual Report of the Board of Regents of the Smithsonian Institution, Washington DC, 1863 (The Smithsonian Institution, Washington, DC, 1863), pp. 207-285.

[2] S. Cohen, F. Plasil, and W. J. Swiatecki, Ann. Phys. (N.Y.) 82, 557 (1974).

[3] G. Gamow, Proc. R. Soc. A 126, 632 (1930).

[4] E. Beaugnon and R. Tournier, Nature (London) 349, 470 (1991).

[5] E. Beaugnon and R. Tournier, J. Phys. III (France) 1, 1423 (1991).

[6] E. Beaugnon, D. Fabregue, D. Billy, J. Nappa, and R. Tournier, Physica (Amsterdam) 294B-295B, 715 (2001).

[7] M. V. Berry and A. K. Geim, Eur. J. Phys. 18, 307 (1997).

[8] M. Sueda, A. Katsuki, M. Nonomura, R. Kobayashi, and Y. Tanimoto, J. Phys. Chem. C 111, 14389 (2007).

[9] See EPAPS Document No. E-PRLTAO-101-065848 for supplementary movies, figures, methods and discussion. For more information on EPAPS, see http://www.aip.org/ pubservs/epaps.html.

[10] R. A. Brown and L.E. Scriven, Proc. R. Soc. A 371, 331 (1980).

[11] T. G. Wang, E. H. Trinh, A.P. Croonquist, and D. D. Elleman, Phys. Rev. Lett. 56, 452 (1986).

[12] T. G. Wang, A. V. Anilkumar, C. P. Lee, and K. C. Lin, J. Fluid Mech. 276, 389 (1994).

[13] P. Aussillous and D. Quéré, Nature (London) 411, 924 (2001).

[14] S. Chandrasekhar, Proc. R. Soc. A 286, 1 (1965).

[15] K. Ohsaka and E.H. Trinh, Phys. Rev. Lett. 84, 1700 (2000).

[16] T. G. Wang, in Advances in Applied Mechanics, edited by J. W. Hutchinson and T. Y. Wu (Academic Press, Inc., San Diego, CA, 1988), Vol. 26, pp. 1-62.

[17] Lord Rayleigh, Proc. R. Soc. London 29, 71 (1879).

[18] N. K. Radyakin, Fluid Dynamics (Springer, NY, 1979), Vol. 14, p. 535.

[19] F. H. Busse, J. Fluid Mech. 142, 1 (1984).

[20] A. Ludu and J. P. Draayer, Phys. Rev. Lett. 80, 2125 (1998).

[21] E.H. Trinh and T. G. Wang, J. Fluid Mech. 122, 315 (1982).

[22] E. H. Trinh, P. L. Marston, and J.L. Robey, J. Colloid Interface Sci. 124, 95 (1988).

[23] Y. Eriguchi and I. Hachisu, Prog. Theor. Phys. 67, 844 (1982).

[24] P. Lacerda and D. C. Jewitt, Astron. J. 133, 1393 (2007).

[25] L. Smarr, Phys. Rev. Lett. 30, 71 (1973).

[26] V. Cardoso and L. Gualtieri, Classical Quantum Gravity 23, 7151 (2006).

[27] V. Cardoso and O. J. C. Dias, Phys. Rev. Lett. 96, 181601 (2006).

[28] N. Schunck, J. Dudek, and B. Herskind, Phys. Rev. C 75, 054304 (2007). 